\title{
Hydromagnetic thin film flow: Linear stability
}

\author{
Mustapha Amaouche, ${ }^{1}$ Hamid Ait Abderrahmane, ${ }^{2, *}$ and Lamia Bourdache ${ }^{1}$ \\ ${ }^{1}$ Department of Physics, Route de Targa-Ouzemour, Béjaia 06000, Algeria \\ ${ }^{2}$ Mathematical and Computer Sciences and Engineering Division, King Abdullah University of Science and Technology, \\ Thuwal, Saudi Arabia
}

(Received 2 December 2012; revised manuscript received 12 April 2013; published 30 August 2013)

\begin{abstract}
This paper deals with the long wave instability of an electroconductor fluid film, flowing down an inclined plane at small to moderate Reynolds numbers, under the action of electromagnetic fields. A coherent second order long wave model and two simplified versions of it, referred to as first and second reduced models (FRM and SRM), are proposed to describe the nonlinear behavior of the flow. The modeling procedure consists of a combination of the lubrication theory and the weighted residual approach using an appropriate projection basis. A suitable choice of weighting functions allows a significant reduction of the dimension of the problem. The full model is naturally unique, i.e., independent of the particular form of the trial functions. The linear stability of the problem is investigated, and the influence of electromagnetic field on the flow stability is analyzed. Two cases are considered: the applied magnetic field is either normal or parallel to the fluid flow direction, while the electric field is transversal. The numerical solution of the Orr-Sommerfeld (OS) eigenvalue problem and those of the depth averaging model are used to assess the accuracy of the reduced models. It is found that the current models have the advantage of the Benney-like model, which is known to asymptote the exact solution near criticality. Moreover, far from the instability threshold, the current reduced models continue to follow the OS solution up to moderate Reynolds numbers, while the averaging model diverges rapidly. The model SRM gives better results than FRM beyond sufficiently high Reynolds numbers.
\end{abstract}

DOI: 10.1103/PhysRevE.88.023028

PACS number(s): 47.15.gm, 47.65.-d

\section{INTRODUCTION}

The stability of fluid films flowing down an inclined plane has received a lot of attention since the pioneering experiments of Kapitza and Kapitza [1]. The instability occurs when the Reynolds number is raised above some critical value which depends on the flow conditions. Then, the flow undergoes a Hopf bifurcation that leads to periodic nonlinear waves which evolve into a variety of patterns, among them the solitary wave structures that play a central role specifically in the long time behavior. Much of the interest in the fluid film flow problem stems from their occurrence in nature and biology; examples include lava flows and eyes' tears. Thin film fluid flows are also encountered in a wide variety of industrial applications, including coating and cooling processes. In some of these applications, the presence of surface waves may be a desirable feature as in the case of cooling films, where they enhance heat and mass transfer [2,3]. Conversely, they are undesirable in the case of coating films, where smooth surfaces are usually required; surface waves alter the finished product and therefore must be avoided [4]. Hence, controlling the formation of waves on film fluid flows is of capital importance, as is their fundamental understanding.

The initial investigations of the stability of fluid film flows began with the studies of Yih [5] and Benjamin [6]. Since then, investigations on the subject all agree that the dominant instability is due to inertial effects and manifests itself at low to moderate Reynolds numbers. It involves free surface undulations with wavelengths much larger than the film depth, except for a slightly inclined plane (less than about $1^{\circ}$ for water)

\footnotetext{
*haitabd@hotmail.com
}

or for fluids with low surface tension. In this condition, in addition to the free surface instabilities, linear analyses have also identified a shear mode instability of the TollmienSchlichting type modified by the presence of free surface. The shear mode instability corresponds to strong shear rates and occurs at rather high Reynolds numbers (about $10^{4}$ ); it may become the primary instability at small angles of tilt. In these extreme conditions, which are beyond the scope of the present study, the free surface is subject to shortwave instability. For small to moderate Reynolds numbers, the range of interest here, the development of asymptotic models is feasible [7,8]. These models describe the initial stage of the flow development and give rise to the so-called Benney equation (BE). Even though the flow variables are all enslaved to the local free surface shape, the BE contains relevant physical mechanisms so that it is potentially capable of accounting for the nonlinear behavior of the flow near criticality. However, the BE loses its physical relevance when inertia becomes important. Indeed, slightly far from the stability threshold, the solution of the BE exhibits nonphysical finite time catastrophic behavior. In order to correct this defect, several improvements were proposed. For example, the regularized procedure developed by Ooshida [9] allows us to prevent the occurrence of finite time blowup but leads to an unrealistic solitary wave solution at moderate Reynolds numbers.

Significant progress in modeling fluid film flow was obtained by associating the fluid flow rate, which becomes a genuine variable just after the wave formation, to the film thickness. This results in two-variable formulation, introduced by Shkadov [10], who used an integral boundary layer (IBL) approach. In spite of its success in describing nonlinear regimes for moderate Reynolds numbers, the IBL does not accurately predict the flow behavior near criticality as the $\mathrm{BE}$ 
does. This defect is due to the fact that some first order terms are omitted in the IBL. A first order coherence is therefore required to correct this defect. The way to do this was proposed, using an approach based on a weighted residual integral boundary layer (WRIBL), by Ruyer-Quil and Manneville in a series of papers [11-13]. This procedure was found to asymptote the BE near criticality and to yield bounded solutions for a larger range of Reynolds numbers. The WRIBL approach was successfully applied to various situations in film flows, such as those involving thermal effects [14,15], two fluid layer flow [16], bottom topography [17], and bottom permeability [18], to name a few.

The main purpose of the present work is to extend the WRIBL to the problem of an electroconducting fluid film flowing under the action of electromagnetic forces and to gain better understanding of the complicated interplay between electromagnetic, surface tension, and gravity forces and its effects on the stability of the flow, which might widen the range of the applications. The interaction of a thin layer of electroconductor fluid, moving under the influence of electromagnetic fields, is of practical importance in several technological applications, such as the casting industry and nuclear reactors. For instance, in belt strip casting magnetic fields are used to produce braking and damping mechanisms [19]. In fusion devices, thin film flows are employed against erosion and thermal loads of the reactor's walls, where the reliability and the efficiency of the protection depend on the flow stability [20]. The orientation of the applied electromagnetic field plays a prominent role in the resulting flow dynamics. Applied alone in the case of perfectly conducting viscous fluid, a normal electric field can destabilize the free surface through a nonlocal force linked to the free surface shape via a Hilbert transform [21-23]. The form of such a nonlocal term has been derived formally in a related horizontal electric field problem in [24-26]. Here, we are concerned with the case where the electric field is applied in the spanwise direction while the magnetic field is in the plane of motion. This configuration was first examined by Korsunsky [27] to complete investigations that were conducted by Lu and Sarma [28] under the action of a transverse uniform magnetic field. These studies revealed that the magnetic field acts to increase the stability and hence can be used to control and even to suppress flow instabilities. Employing both the long wave asymptotic approach, leading to a Kuramoto-Sivashinsky equation, and the IBL approach, Korsunsky [27] found that depending on its orientation, the electric field is either stabilizing or destabilizing, whereas the magnetic field is always stabilizing. The IBL approach was also used in $[29,30]$ to examine the combined effects of spanwise electric and transverse magnetic fields. These two studies confirmed the findings of Korsunsky [27] with respect to the influence of electric and magnetic fields on the flow stability. It should be noted that, as in the ordinary film flow, neither the long wave asymptotic expansion nor the IBL approach allows an accurate description of the stability analysis for both small and moderate Reynolds numbers. Introducing a more realistic velocity profile than the one used in the IBL approach, it is expected that the WRIBL removes the drawbacks described above, includes lower orders of the electromagnetic force, and accordingly provides an accurate description of the flow for small to moderate Reynolds

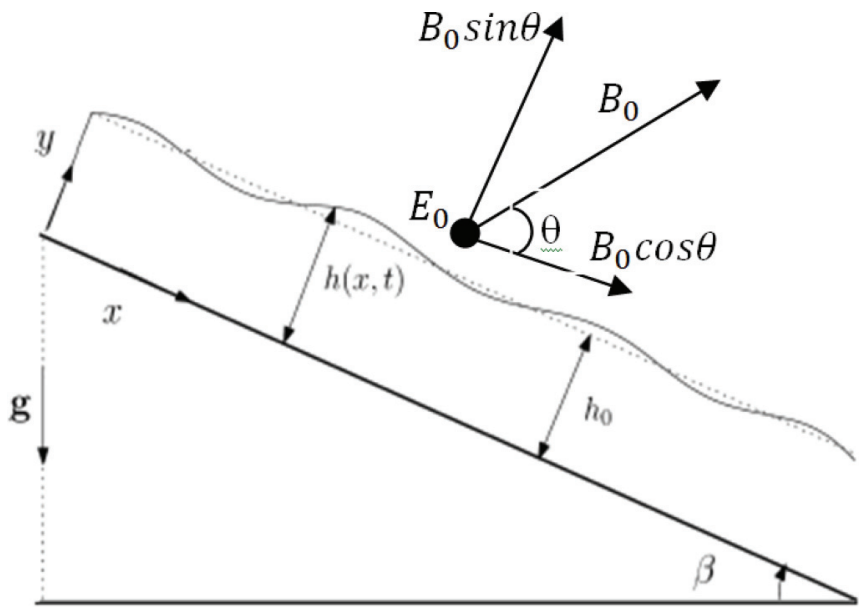

FIG. 1. (Color online) Schematic of the physical problem.

numbers. This is the objective of the current analysis, which is organized as follow. In Sec. II, the problem is formulated and expressed, up to second order, in the framework of lubrication theory. A way to derive nonlinear evolution equations via a weighted residual approach is outlined in Sec. III. Because the full model has little practical use, more tractable models of reduced dimensionality are proposed in Sec. IV. Linear stability results are presented in Sec. V in comparison with OrrSommerfeld (OS) analysis. Concluding remarks are finally given in Sec. VI.

\section{GOVERNING EQUATIONS AND BASIC FLOW}

We consider the stability of a two-dimensional gravity driven film flow down an infinitely long flat plate in the presence of an electromagnetic field. The plate is nonconducting and inclined at an angle $\beta$ with respect to the horizontal. The liquid, of constant density $\rho$ and kinematic viscosity $v$, is assumed to be a perfect conductor with conductivity $\sigma$ and magnetic permeability $\mu_{0}$, and the surrounding passive gas is of negligible density and conductivity. The surface tension between the liquid and the gas is $\gamma$, and the acceleration due to gravity is denoted by $g$. A rectangular coordinate system $(x, y, z)$ is adopted, with $x$ and $y$ measuring distances downstream and perpendicular to the plate, respectively, and $z$ being in the spanwise direction (see the physical model depicted in Fig. 1). The film thickness is given by $y=h(x, t)$, where $h_{0}$ is its constant value in the base state. The applied electric field is $\boldsymbol{E}_{\mathbf{0}}=\left(0,0, E_{0}\right)$, and for the completeness of the mathematical formulation we take the magnetic field in the plane of motion $\boldsymbol{B}_{\mathbf{0}}=B_{0}(\cos \theta, \sin \theta, 0)$.

Within the usual magnetohydrodynamics (MHD) approximations, the governing equations describing the conservation of mass, momentum, magnetic flux, and the advectiondiffusion of the magnetic field read

$$
\nabla \cdot \boldsymbol{v}=0
$$

$$
\frac{D \boldsymbol{v}}{D t}=-\frac{1}{\rho} \nabla\left(p+\frac{\mathbf{B}^{2}}{2 \mu_{0}}\right)+\mathbf{g}+v \nabla^{2} \boldsymbol{v}+\frac{1}{\rho \mu_{0}}(\mathbf{B} \cdot \nabla) \mathbf{B},
$$




$$
\begin{gathered}
\nabla \cdot \boldsymbol{B}=0, \\
\frac{D \mathbf{B}}{D t}=(\mathbf{B} \cdot \nabla) \boldsymbol{v}+\frac{1}{\mu_{0} \sigma} \nabla^{2} \mathbf{B},
\end{gathered}
$$

where $\frac{D}{D t}$ stands for the two-dimensional material derivative, $\mathbf{B}$ is the total magnetic field, and $\mathbf{v}=(u, v, 0)$ is the velocity field. The Lorentz force includes two parts: the irrotational part, which acts as a pressure force $\left(-\frac{1}{\rho} \nabla \frac{\mathbf{B}^{2}}{2 \mu_{0}}\right)$, and a rotational part $\left[\frac{1}{\rho \mu_{0}}(\mathbf{B} \cdot \nabla) \mathbf{B}\right]$. The induction equation (4) results from the combination of the following classical Maxwell equations:

$$
\begin{gathered}
\nabla \times \mathbf{E}=-\frac{\partial \mathbf{B}}{\partial t}, \\
\mu_{0} \boldsymbol{j}=\nabla \times \mathbf{B}, \\
\boldsymbol{E}=\boldsymbol{j} / \sigma-\boldsymbol{v} \times \boldsymbol{B},
\end{gathered}
$$

where $\mathbf{j}$ and $\mathbf{E}$ are the current density and the electric field, respectively. These equations are subject to appropriate boundary conditions. Instead of setting them in the general case, these conditions are specified once the problem is simplified in the framework of the lubrication approximation. For that purpose we first introduce the following dimensionless variables: $t^{*}=$ $\epsilon \frac{u_{0}}{h_{0}} t, x^{*}=\epsilon \frac{x}{h_{0}}, y^{*}=\frac{y}{h_{0}}, u^{*}=\frac{u}{u_{0}}, v^{*}=\epsilon \frac{v}{u_{0}}$, where $\epsilon$ is the film parameter accounting for the smallness of the temporal and streamwise variations compared to the transverse gradients $\left(\partial_{y} \sim 1, \partial_{x} \sim \epsilon, \partial_{t} \sim \epsilon\right)$ and $u_{0}=g h_{0}^{2} \sin \beta / 3 v$ is the depth averaged velocity of the classical Nusselt flow. Then, dropping the asterisks for convenience, the dimensionless coupled MHD equations governing the film flow can be reduced up to $O\left(\epsilon^{2}\right)$ (see Appendix A for derivation):

$$
\begin{aligned}
& \operatorname{Re} \epsilon\left(u_{t}+u u_{x}+v u_{y}\right) \\
&= u_{y y}+3-\mathrm{Ha}^{2} \sin \theta(\delta+u \sin \theta) \\
&+\epsilon\left\{W h_{x x x}-3 \cot \beta h_{x}+\mathrm{Ha}^{2} \cos \theta\right. \\
&\left.\times\left[\sin \theta\left(2 v-\left.v\right|_{h}\right)+h_{x}\left(\delta+\left.\sin \theta u\right|_{h}\right)\right]\right\} \\
&+\epsilon^{2}\left\{2 u_{x x}+\left(\left.u_{x}\right|_{h}\right)_{x}+\mathrm{Ha}^{2} \cos ^{2} \theta\right. \\
&\left.\times\left[\int_{h}^{y} v_{x} d y-\left.v\right|_{h} h_{x}\right]\right\}, \\
& u_{x}+v_{y}=0,
\end{aligned}
$$

which represent conservation of momentum and mass, respectively. It should be noted that the magnetic and electric fields are scaled by $B_{0}$ and $E_{0}$, respectively. Equations (8) and (9) must be solved, subject to (i) impermeability and no slip conditions on the solid wall,

$$
\left.u\right|_{y=0}=\left.v\right|_{y=0}=0,
$$

and (ii) impermeability and tangential stress balance on the free surface,

$$
\begin{gathered}
\left.v\right|_{h}=h_{t}+\left.u\right|_{h} h_{x}, \\
\left.u_{y}\right|_{h}=\epsilon^{2}\left(\left.4 h_{x} u_{x}\right|_{h}-\left.v_{x}\right|_{h}\right) .
\end{gathered}
$$

As can be seen from (8), the flow dynamics is controlled by four parameters, namely, the Reynolds number $\operatorname{Re}=u_{0} h_{0} / v$, the Hartmann number $\mathrm{Ha}=B_{0} h_{0} \sqrt{\sigma / \rho \nu}$, the electric number $\delta=E_{0} / u_{0} B_{0}$, and the capillary number $W=\epsilon^{2} \mathrm{ReWe}$, with $\mathrm{We}=\sigma / \rho h_{0} u_{0}^{2}$ being the classical Weber number. The term factored by $\mathrm{Ha}^{2}$ is the Lorentz force, which contains two parts. The first part is the one multiplied by $\delta$; it is produced by the magnetic field induced by the conductive current. The second part is generated by the magnetic field induced by the convective current. The contribution of the Lorentz force to the flow stability depends on the orientation of the electric field and the direction of the magnetic field. In the following, the orientation of the electric field is considered either positive or negative, while the orientation of the magnetic field is kept positive. Under this condition, the work of the conductive part of the Lorentz force depends on the orientation of the electric field (the sign of $\delta$ ). When the magnetic field is perpendicular $(\sin \theta=1)$, the Lorentz force intervenes as gravity at order zero with respect to $\epsilon$, while it intervenes at first and second orders when the magnetic field is parallel $(\sin \theta=0)$.

The basic velocity field is $x$ directional and is given by

$u_{b}(y)=\left\{\frac{3}{\mathrm{Ha}^{2} \sin ^{2} \theta}-\frac{\delta}{\sin \theta}\right\}\left\{1-\frac{\cosh \mathrm{Ha} \sin \theta(y-1)}{\cosh (\mathrm{Ha} \sin \theta)}\right\}$,

which is the solution of the basic equation resulting from (8) by dropping terms factored by $\epsilon$. The depth averaged value of $u_{b}(y)$ reads

$$
u_{m b}=\left\{\frac{3}{\mathrm{Ha}^{2} \sin ^{2} \theta}-\frac{\delta}{\sin \theta}\right\}\left\{1-\frac{\tanh (H a \sin \theta)}{\mathrm{Ha} \sin \theta}\right\} .
$$

In order to illustrate the influence of electromagnetic effect on the mean flow we show in Fig. $2 u_{m b}$ versus Ha $\sin \theta$ for some representative values of $\delta / \sin \theta$. It is shown that, for all positive values of $\delta / \sin \theta$, the mean velocity is a monotonically decreasing function of Ha $\sin \theta$. Moreover, the flow may change direction for a given $\delta / \sin \theta$ if the Hartmann number is high enough $\left(\mathrm{Ha}^{2}>3 / \delta \sin \theta\right)$. Hence, in the case where the electric field is oriented in the positive $z$ direction, the two parts of the Lorentz force both act against gravity. When the electric field is oriented in the negative $z$ direction, the associated part of the Lorentz force accelerates the mean flow. Beyond a sufficiently high level of electric field, the combined effects of gravity and Lorentz electric forces overcome the

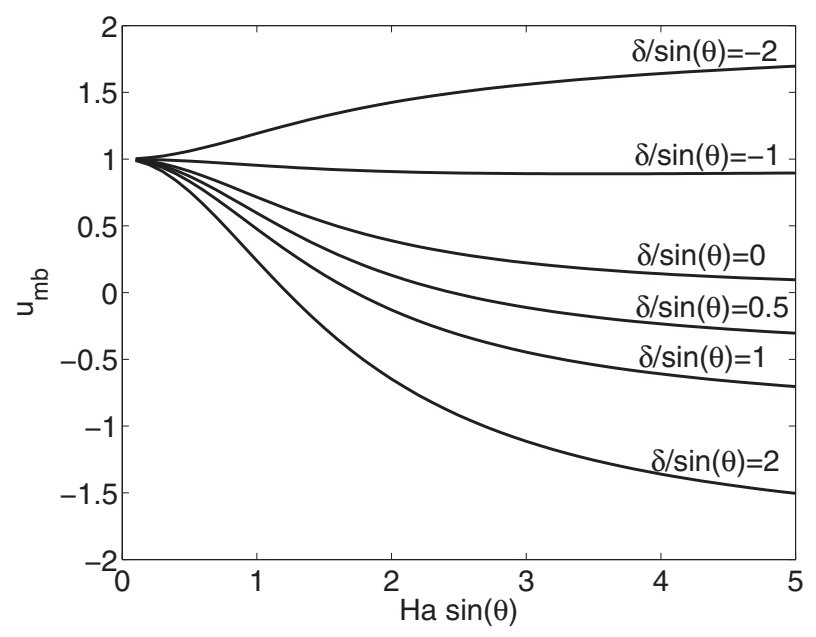

FIG. 2. Mean flow velocity vs Ha $\sin \theta$ for some values of the electric parameter. 
Lorentz force generated by convection. Now, let us observe that the kinematic condition (11) can be rewritten owing to (9) in a global form as

$$
q_{x}+h_{t}=0,
$$

where $q=\int_{o}^{h} u d y$ is the flow rate in the streamwise direction.

\section{MODELING THE WAVY FILM FLOW}

In the framework of shallow water theory, the streamwise velocity is expanded in a power series of the shallowness parameter $\epsilon$. Up to $O\left(\epsilon^{2}\right)$ this reads

$$
u(x, y, t)=u_{0}^{*}+\epsilon u_{1}^{*}+\epsilon^{2} u_{2}^{*}+O\left(\epsilon^{3}\right),
$$

where $u_{1}^{*}$ and $u_{2}^{*}$ are referred to as the first and second order corrections to the leading order term $u_{0}^{*}$. In applying the weighted residual method, these corrections are in turn expanded in some complete set of trial functions which depend on the physical problem under consideration. For the sake of simplification, the modeling will be focused only on the particular cases $\theta=\frac{\pi}{2}$ and $\theta=0$, corresponding to normal and longitudinal magnetic fields, respectively.

\section{A. Perpendicular magnetic field}

In this case, the basic velocity $u_{b}$ is deduced from (13) by putting $\sin \theta=1$; this gives $u_{b}=\frac{1}{\mathrm{Ha}^{2}}\left[3-\delta \mathrm{Ha}^{2}\right][1-$ $\left.\frac{\cosh \mathrm{Ha}(y-1)}{\cosh \mathrm{Ha}}\right]$. Similar to $u_{b}, u_{0}^{*}$ is searched in the form $u_{0}^{*}=a_{0}(x, t) f_{0}(\xi)$, where $\xi=y-h(x, t), f_{0}(\xi)=\frac{1}{\mathrm{Ha}^{2}}(1-$ $\left.\frac{\cosh \mathrm{Ha} \xi}{\cosh \mathrm{Ha} h}\right)$, and $a_{0}(x, t)$ is an unknown amplitude. Since the flow rate appears in (15) as a genuine variable like the film depth, it is quite natural to eliminate $a_{0}(x, t)$ in favor of these two variables. This can be done by integrating (16) through the film depth; one obtains

$$
\begin{aligned}
a_{0}(x, t)= & \frac{1}{\int_{0}^{h} f_{0} d y}\left\{q(x, t)-\epsilon \int_{0}^{h} u_{1}^{*} d y-\epsilon^{2} \int_{0}^{h} u_{2}^{*} d y\right\} \\
& +O\left(\epsilon^{3}\right) .
\end{aligned}
$$

Expansion (16) then takes the form

$$
u(x, y, t)=u_{0}+\epsilon u_{1}+\epsilon^{2} u_{2}+O\left(\epsilon^{3}\right),
$$

where

$$
\begin{aligned}
& u_{0}=\frac{q(x, t)}{\int_{0}^{h} f_{0} d y} f_{0}(\xi), \\
& u_{1}=u_{1}^{*}-\frac{\int_{0}^{h} u_{1}^{*} d y}{\int_{0}^{h} f_{0} d y} f_{0}(\xi), \\
& u_{2}=u_{2}^{*}-\frac{\int_{0}^{h} u_{2}^{*} d y}{\int_{0}^{h} f_{0} d y} f_{0}(\xi) .
\end{aligned}
$$

The above formulation shows that the flow rate is entirely contained in the leading order term $u_{0}$ while $\int u_{1} d y=$ $\int u_{2} d y=0$. Remember that the primary objective of the present work is to attempt to lower the dimensionality of the problem by eliminating from the calculation the second order correction $u_{2}$. This is feasible under some conditions that will be explained below. The explicit form of $u_{2}$ then would not be useful. An exact expansion for $u_{1}$ is, however, of capital importance in order to ensure the second order coherence of the model. It can be obtained by equating first order terms in (8) where the above expressions for $u$ and $u_{0}$ are inserted. The result is the following equation for $u_{1}$ :

$$
u_{1 y y}-\mathrm{Ha}^{2} u_{1}=\operatorname{Re}\left[u_{0 t}+u_{0} u_{0 x}+v_{0} u_{0 y}\right],
$$

subject to $\left.u_{1}\right|_{y=0}$ and $\left.u_{1 y}\right|_{y=h}=0$. The solution for this boundary value problem, satisfying the global constraint $\int u_{1} d y=0$, is

$$
u_{1}(x, y, t)=\operatorname{Re}\left[a(x, t) f_{1}(\xi)+b(x, t) f_{2}(\xi)\right],
$$

where $a$ and $b$ are unknown functions to be determined. The trial functions $f_{1}$ and $f_{2}$ are found to be

$$
\begin{aligned}
& f_{1}(\xi)=f_{1}^{*}(\xi)+\delta_{1}(x, t) f_{0}(\xi), \\
& f_{2}(\xi)=f_{2}^{*}(\xi)+\delta_{2}(x, t) f_{0}(\xi),
\end{aligned}
$$

where $f_{1}^{*}, f_{2}^{*}, \delta_{1}(x, t)$, and $\delta_{2}(x, t)$ are given in Appendix B. Hence, provided that $u_{2}$ does not participate in the calculation as it is already emphasized, the film dynamics can be described by only four unknown variables, namely, $h, q, a$, and $b$. It is important to notice that the trial function $f_{1}$ is fixed once for all due to the constraint $\int u_{1} d y=0$, while $f_{2}$ may still be combined with $f_{1}$ so as to orthogonalize the resulting function with $f_{0}$ or $f_{1}$. At this stage, this operation is not necessary since it generates a too cumbersome function; $f_{2}$ is then kept unchanged for the sake of simplification. In addition to (15), three other equations are therefore required to describe the space and time evolution of the fields $q, h, a$, and $b$. These equations may be obtained by forcing the residuals to vanish in the mean sense, i.e., by setting integrals of the residuals equal to zero.

$$
\begin{gathered}
\int_{0}^{h}\left\{\operatorname{Re} \epsilon\left(u_{t}+u u_{x}+v u_{y}\right)-u_{y y}+\mathrm{Ha}^{2}(u+\delta)-3\right. \\
\left.-\epsilon\left(W h_{x x x}-3 \cot \beta h_{x}\right)-\epsilon^{2}\left[2 u_{x x}+\left(\left.u_{x}\right|_{h}\right)_{x}\right]\right\} \phi_{i} d y=0 \\
i=0,1,2
\end{gathered}
$$

where $\phi_{i} i=1,2,3$ are some suitable functions to be specified below. First, one can observe that the spectral coefficients of the correction $u_{2}$ would appear only through the transverse diffusion term $u_{y y}$ along with the magnetic force $\mathrm{Ha}^{2} u$ since all the others are at least of order $\epsilon$. Their elimination procedure can be made clearer by rewriting the integral $\int_{0}^{h}\left(u_{y y}-\mathrm{Ha}^{2} u\right) \phi_{i} d y$ in the form

$$
\begin{aligned}
\int_{0}^{h}\left(u_{y y}-\mathrm{Ha}^{2} u\right) \phi_{i} d y= & \left.u_{y} \phi_{i}\right|_{0} ^{h}-\left.u \phi_{i y}\right|_{0} ^{h} \\
& +\int_{0}^{h} u\left(\phi_{i y y}-\mathrm{Ha}^{2} \phi_{i}\right) d y .
\end{aligned}
$$

Now, one can see that this integral can be rendered free from $u_{2}$ by properly choosing the weighting functions. In this perspective these functions are required to first satisfy the homogeneous boundary conditions $\left.\phi_{i}\right|_{0}=\left.\phi_{i y}\right|_{h}=0$ because of the wall condition $(u=0)$ and since $\left.u_{y}\right|_{h}=O\left(\epsilon^{2}\right)$. In addition to these conditions, the quantity $\phi_{i y y}-\mathrm{Ha}^{2} \phi_{i}$ must be either constant or orthogonal in the sense of $L^{2}(0, h)$ to the projection basis of $u_{2}$. The latter is not explicitly required for the calculation up to $O\left(\epsilon^{2}\right)$, but it can be derived in a similar way as for $u_{1}$, and most importantly, it can be modified to be 
orthogonal to at least two arbitrarily functions, say $f_{0}$ and $f_{1}$. This property is taken advantage of to define the weighting functions in the following way. When $\phi_{0}=f_{0}, \phi_{1}$ and $\phi_{2}$ are required to satisfy the equations

$$
\begin{aligned}
\phi_{1}^{\prime \prime}-\mathrm{Ha}^{2} \phi_{1} & =f_{0}, \\
\phi_{2}^{\prime \prime}-\mathrm{Ha}^{2} \phi_{2} & =f_{1} ;
\end{aligned}
$$

along with the above homogeneous boundary conditions, one obtains $\phi_{1}$ and $\phi_{2}$ in the form

$$
\begin{aligned}
& \phi_{1}=-\left(\frac{1}{\cosh \mathrm{Ha} h} f_{1}^{*}+\frac{1}{\mathrm{Ha}^{2}} f_{0}\right), \\
& \phi_{2}=\frac{1}{2} f_{2}^{*}+\frac{\delta_{1}}{\mathrm{Ha}^{2}} \phi_{1}-\frac{h}{2 \mathrm{Ha}} \tanh \mathrm{Ha} h
\end{aligned}
$$

Finally, the residual equations may be cast in the form

$$
\operatorname{Re} \mathcal{A}(q, a, b)^{t}=(Q, A, B)^{t} .
$$

The entries of the matrix $\mathcal{A}$ along with the components $Q, A$, and $B$ are too cumbersome to be included here.

\section{B. Longitudinal magnetic field}

When the magnetic field is oriented in the streamwise direction, the momentum conservation equation reduces, by setting $\sin \theta=0$, to

$$
\begin{aligned}
\operatorname{Re} \epsilon & \left(u_{t}+u u_{x}+v u_{y}\right) \\
= & u_{y y}+3+\epsilon\left\{W h_{x x x}+h_{x}\left(\delta \mathrm{Ha}^{2}-3 \cot \beta\right)\right\} \\
& +\epsilon^{2}\left\{2 u_{x x}+\left(\left.u_{x}\right|_{h}\right)_{x}+\mathrm{Ha}^{2}\left[\int_{h}^{y} v_{x} d y-\left.v\right|_{h} h_{x}\right]\right\},
\end{aligned}
$$

which shows that the produced Lorentz force has no effect on the basic flow but acts when the latter is perturbed. That action first occurs at $O(\epsilon)$ via the modification of the streamwise gravitational pressure drop, which is either lowered or augmented, depending on the orientation of the electric field. At $O\left(\epsilon^{2}\right)$, the change is caused by the convective part of the Lorentz force and turns on the streamwise diffusive terms. Then the basic solution is the classical Nusselt flow, which can be deduced from (13) by taking the limit $\mathrm{Ha}^{2} \sin \theta \rightarrow 0$; this yields $u_{b}=3\left(y-\frac{1}{2} y^{2}\right)$. As a consequence, the wavy film problem remains, at the procedure level, similar to the classical case [14] which can be deduced as a limit, when $\mathrm{Ha}^{2} \sin \theta \rightarrow 0$, of the general problem under consideration.

\section{REDUCED MODELS}

As mentioned above the full size second order model is too cumbersome and accordingly of little practical interest. Hence, reduced models which, however, would contain the relevant dynamic characteristics of the full size model are to be desired. Those simplified models should asymptote Benney's single equation near criticality but without its drawbacks and should accurately describe the flow dynamics up to at least moderate Reynolds numbers. It can be shown that only the fields $q$ and $h$ will play a pertinent role at the initial stage of the instability, while the fields $a$ and $b$ follow their dynamics. First, let us observe that due to the special choice of test functions, the fields $a$ and $b$ do appear in the first residual equation only through the second order inertial term. The latter is associated with the advection, by the base flow, of the first order velocity correction. Hence, one can eliminate $a$ and $b$ in that equation since these fields are already known up to the first order. Indeed, equating first order terms in (1) provides

$$
\begin{aligned}
& a(x, t)=\alpha_{1} q_{x}+\alpha_{2} h_{x}+\alpha_{3} q_{t}, \\
& b(x, t)=\beta_{1} q_{x}+\beta_{2} h_{x},
\end{aligned}
$$

where the coefficients $\alpha$ and $\beta$ are given in Appendix B. It is worth noting that these expressions, as functionals of $h$ and $q$, are not unique because of their dependence on the test functions. Another set of test functions would lead to formally different expressions. The corresponding first order correction of the velocity profile, however, remains unchanged, irrespective of the choice of trial functions, provided that these functions satisfy the conditions stated previously. Another way to obtain appreciable simplifications consists of assuming fields $a$ and $b$ to be at least $O\left(\epsilon^{2}\right)$. Hence, their adiabatic elimination in the first residual equation allows us to greatly simplify the formulation, which, however, captures all the physical mechanisms. In fact, the resulting model can be deduced from the previous one by simply dropping the inertial term proportional to $\mathrm{Re}^{2}$. These two reduced models, here referred to as the first and second reduced models, respectively (FRM and SRM), are given as Supplemental Material [31]. We notice that in the limit $\mathrm{Ha}=0$ the two reduced models give those obtained in [32] for $n=1$.

\section{LINEAR STABILITY AND APPLICATIONS}

In this section, the models presented above are applied in order to examine the influence of magnetic and electric fields on the linear response to a sinusoidal perturbation of the film. We assess the accuracy of these models by comparing their linear spectrum to that corresponding to the OS numerical analysis (see Appendix C). We first present the results of the numerical resolution of the eigenvalue problem (C3)-(C6) and depict the influence of electromagnetic fields on the flow stability. For the sake of clarity the presented results are restricted to two directions of the magnetic field with respect to the flow direction $\left(\theta=0\right.$ and $\left.\theta=\frac{\pi}{2}\right)$. The electrical field is oriented either in a positive or negative direction, while the magnetic field is always oriented in the positive direction. The surface tension parameter and the inclination angle of the plane were kept constant and fixed to $W=10$ and $\beta=\frac{\pi}{2}$, respectively.

The marginal stability curves, which separate stable (above) and unstable (below) regions in the $\alpha$-Re space, are displayed in Figs. 3(a)-3(d). The induced Lorentz force contains two parts, namely, the conductive and the convective parts. The conductive part is the one produced by the magnetic field induced by the conductive current, while the convective part is generated by the magnetic field induced by the convective current. In the absence of the electrical field, the Lorentz force reduces to its convective part, and this force opposes the cause which has given birth, i.e., the fluid flow. The convective part work to stabilize the flow; see Figs. 3(a) and 3(b). In fact, in the case of perpendicular magnetic field, the Lorentz force results from the interaction of the magnetic field with the streamwise component of the velocity. This force, which is of order 1 , is 

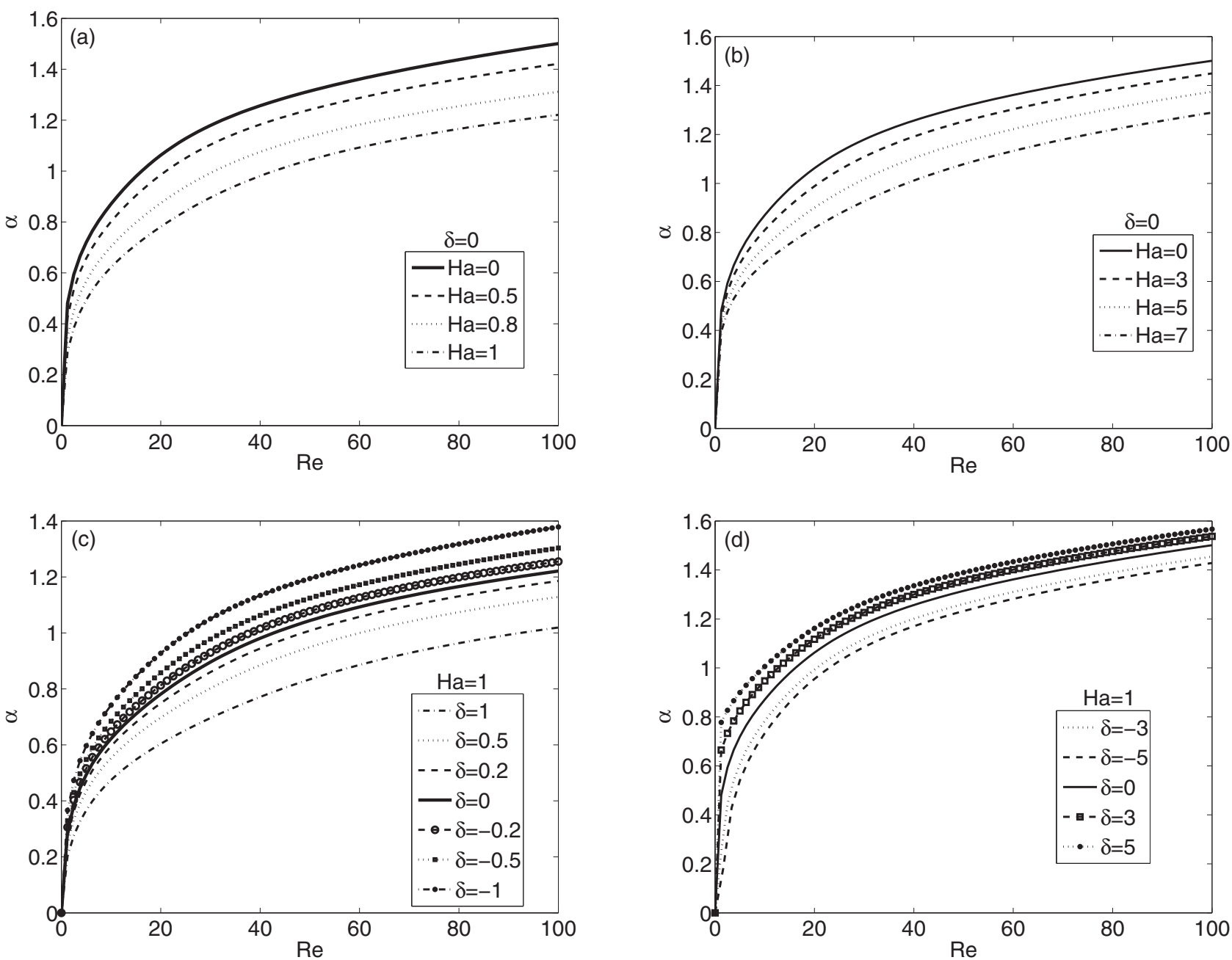

FIG. 3. Marginal wave number vs Reynolds number: influence of electromagnetic fields on the marginal stability of the flow for (a) and (c) perpendicular magnetic field $(\theta=\pi / 2)$ and (b) and (d) longitudinal magnetic field $(\theta=0) . \beta=\pi / 2$.

oriented upstream and works against the destabilizing gravity force [Fig. 3(a)]. In the case of parallel magnetic field, the Lorentz force results from the interaction of the magnetic field and the vertical component of the perturbed velocity field. This force, which is of order $\epsilon$, acts as a supplementary pressure and opposes any free surface elevation [Fig. 3(b)]. One should apply stronger parallel magnetic field to achieve a stabilizing effect similar to that of perpendicular magnetic field. The lines of the magnetic field act as elastic strings; they pull up the streamwise flow in the case of perpendicular field and oppose any free surface elevation in the normal direction in the case of parallel magnetic field. As the field strength increases, the elastic strings stiffen, and the opposition to the fluid motion becomes stronger.

When the electric field is applied, a conductive part of the Lorentz force adds to the convective part. In the case where the magnetic field is perpendicular and the electric field is oriented in the positive $z$ direction, the conductive part of the Lorentz force is oriented upstream. It works, like the convective part, to oppose the destabilization of the flow by the gravity force; see Fig. 3(c). Conversely, when the orientation of the electric field is in the negative $z$ direction, the conductive part of the
Lorentz force is aligned downstream. It works now in the sense of the acceleration and the destabilization of the fluid flow [Fig. 3(c)]. The portrait is opposite when the magnetic field is parallel. In fact, when the electric field is oriented in the negative $z$ direction, the conductive part of the Lorentz forces acts, as a pressure, in the direction perpendicular to the fluid flow. Its effect is added to that of the convective part to oppose any free surface elevation [Fig. 3(d)]. In contrast, when the orientation of the electric field is in the positive $z$ direction, the conductive part of the Lorentz force works against a pressure and the convective part to promote deformation of the free surface; see Fig. 3(d). Therefore, the electrical field can have either a stabilizing or destabilizing role. This depends on its orientation, and the direction of the magnetic field considered here is always positive.

The influence of the electromagnetic fields on the growth rate of the flow perturbation is depicted in Figs. 4(a)-4(d). These results are in accordance with those in Figs. 3(a)-3(d); i.e., they confirm the stabilizing effect of the magnetic field and the dependence of the role of the electric field (destabilizing or stabilizing) on its orientation. The first striking observation is that the wave number corresponding to the fast growing 

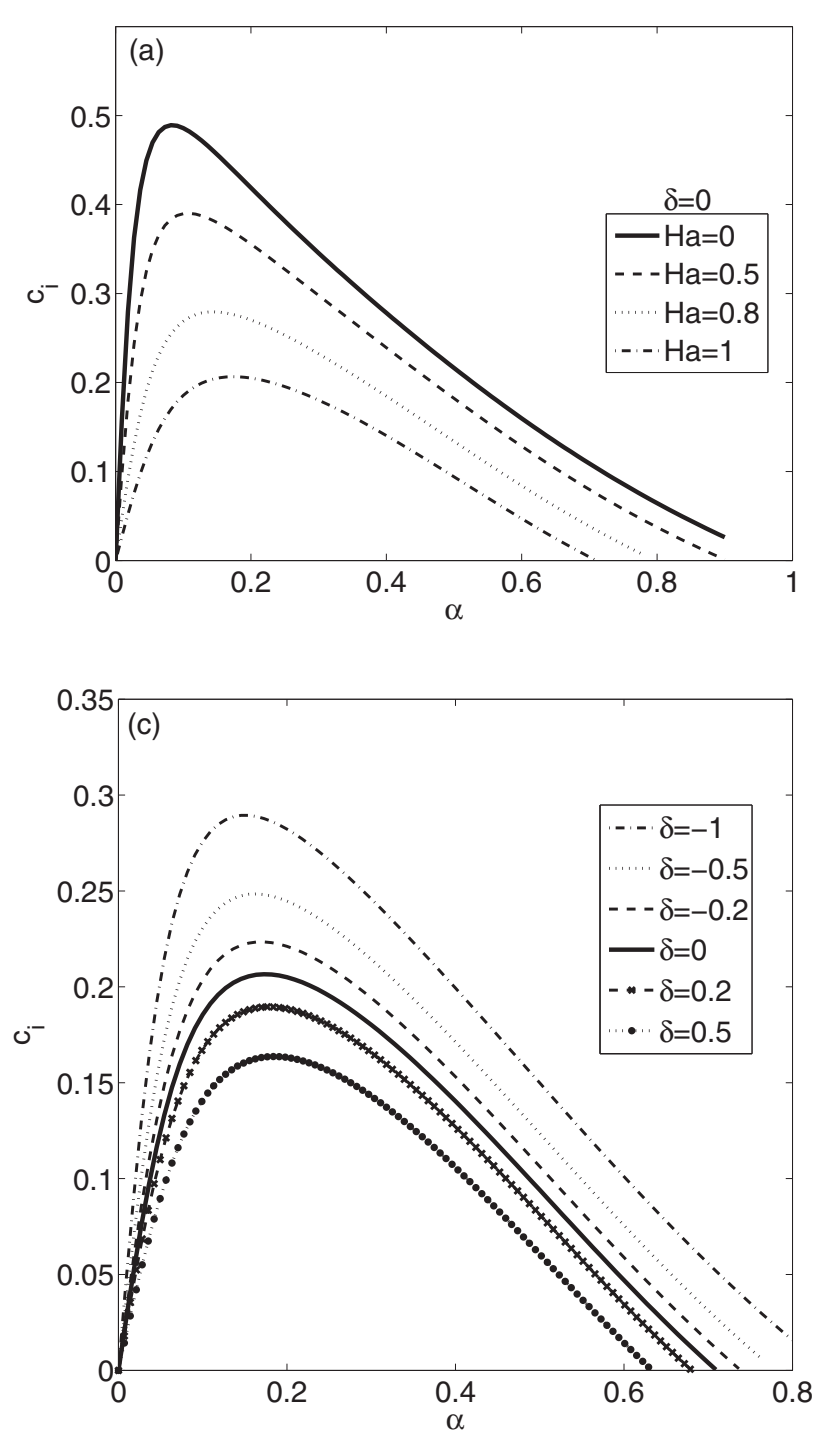
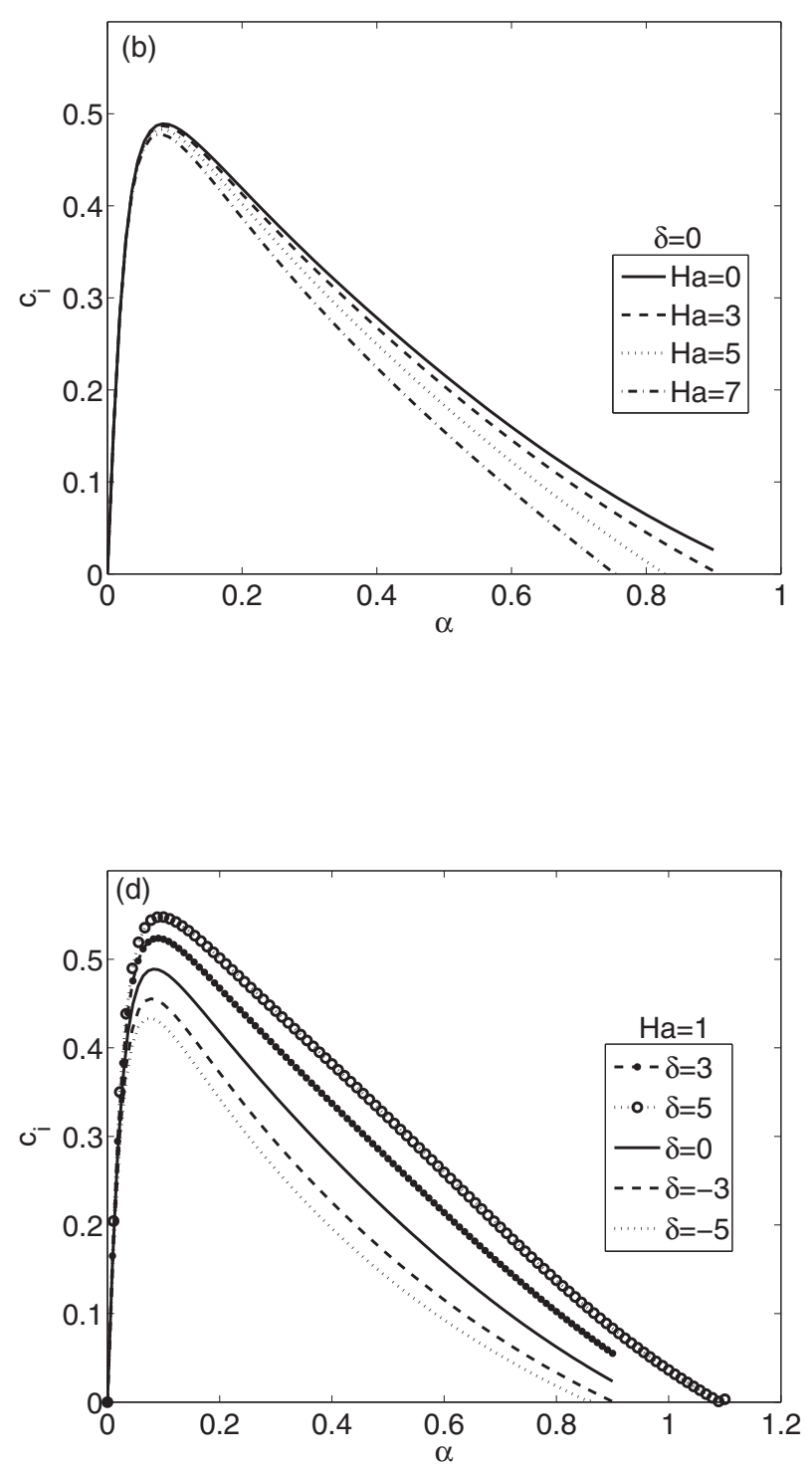

FIG. 4. Growth rate vs wave number: influence of electromagnetic fields on the instability growth rate for (a) and (c) perpendicular magnetic field $(\theta=\pi / 2)$ and $(\mathrm{b})$ and $(\mathrm{d})$ and longitudinal magnetic field $(\theta=0)$. $\mathrm{Ha}=1, \beta=\pi / 2$, and $\operatorname{Re}=15$.

perturbation remains almost constant $(\alpha \approx 0.15)$. The second observation is that even if it is intense, a parallel magnetic field has a weak influence on the growth rate of the perturbation; its influence on the fast growing perturbation is quasinull [Fig. 4(b)].

The results from the numerical solution of the OS problem are used as a benchmark case to assess the accuracy, at the linear stage, of the reduced models presented in Sec. III. The results of the linear stability of the basic flow obtained via FRM are compared to those provided by the solution of the OS eigenvalue problem, SRM, and integral (IM) models. The latter model results from the application of the Karman-Polhausen IBL theory, while the SRM is obtained by simply omitting the first correction $u_{1}$ in the second order equation (A24). Figure 5 compares the marginal stability curves provided by FRM to the numerical solution of the OS problem in both situations, perpendicular $\left(\theta=\frac{\pi}{2}\right)$ and parallel magnetic fields $(\theta=0)$. Figure 5 show that the prediction of FRM agrees well with the numerical results of the OS eigenvalue problem up to Reynolds number $\mathrm{Re} \approx 20$. After that the reduced model experiences a rapid divergence from the exact neutral curve OS. Figure 5 shows also that SRM predicts fairly well the exact neutral curve OS up to $\operatorname{Re} \approx 13$. Beyond this value the SRM starts to diverge slowly while remaining asymptotic and following the same trend as the OS solution. Figure 5 also indicates that the neutral stability curves predicted by the depth IM proposed by Korsunsky [27] and Mukhopadhyay et al. [29] fail to depicted the neutral stability curve OS. In fact, this result was expected because the depth integral model reduces to the Shkadov model [10] and it is a well known drawback of Shkadov's model. The second well known drawback of depth IM is the prediction of the correct stability threshold (critical Reynolds number). The evolution of the critical Reynolds number with the Hartmann number for different value of the parameter $\delta$ obtained with FRM and IM is shown in Fig. 6. Figure 6 indicates that the critical Reynolds number, predicted 

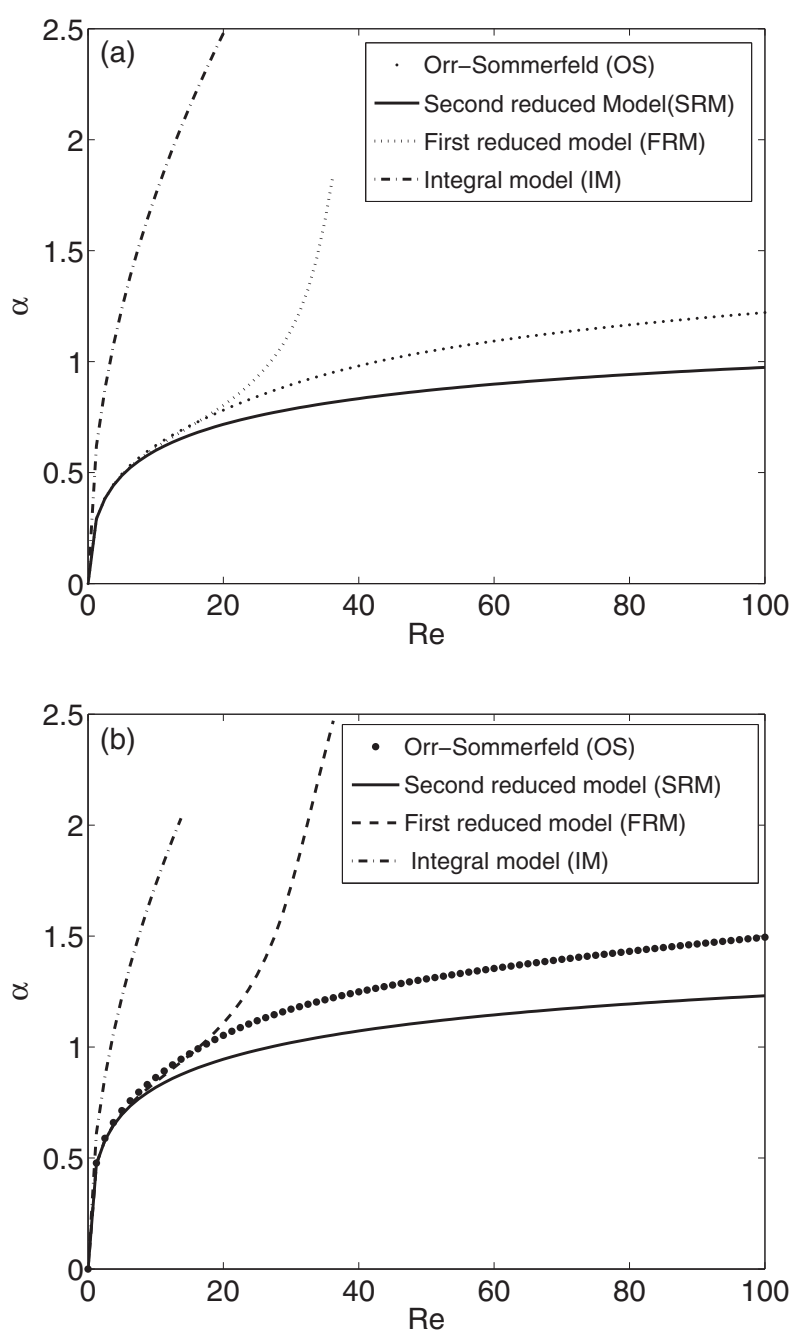

FIG. 5. Comparison of the models' predictions with the OrrSommerfeld solution: (a) the magnetic field is perpendicular; (b) the magnetic field is parallel. In (a) and (b) $\beta=\pi / 2$ and $\delta=0$.

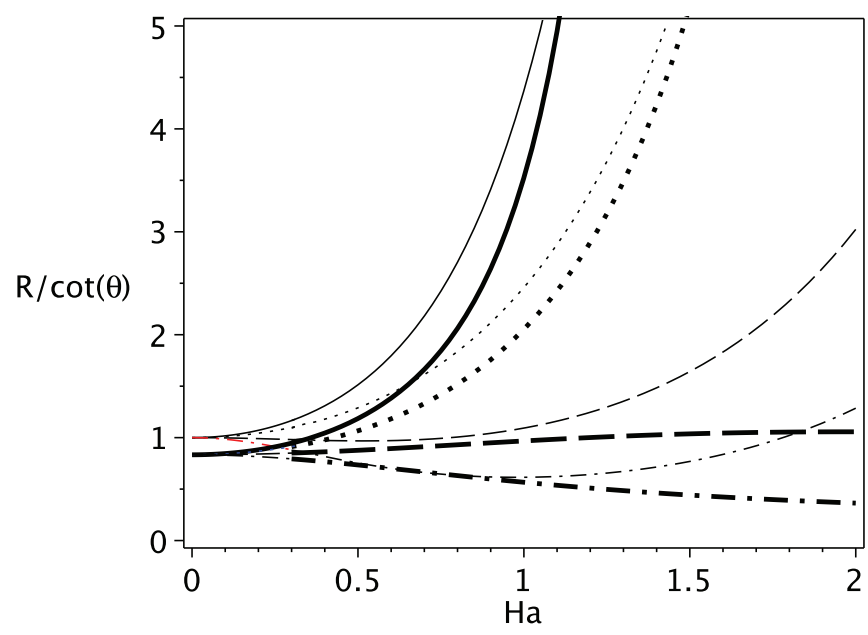

FIG. 6. (Color online) Comparison of the FRM and IM predictions for the critical Reynolds number. The magnetic field is perpendicular. Thin lines are from the IM model, while thick lines are from the FRM model. Solid line, $\delta=0$; dotted line, $\delta=-1$; dashed line, $\delta=-3$; dot-dashed line, $\delta=-5$. by FRM for the onset of instability, reduces to the correct critical value $\operatorname{Re}_{c}=\frac{5}{6} \cot \beta$ of the hydrodynamic case, while the IM model predicts $\operatorname{Re}_{c}=\cot \beta$.

\section{CONCLUSIONS}

In this study we have derived models of reduced dimensionality to investigate the stability of an electroconductor fluid film flowing down an inclined plane. The OS eigenvalue problem is also formulated and solved. Due to the little practical use of the second order coherent analysis, two reduced models are proposed; they both predict, with a fairly good accuracy, the exact solution of OS equations up to about $\mathrm{Re}=20$. Beyond this value of Re the model FRM diverges rapidly, while the model SRM continues to be in agreement with the exact solution up to Reynolds numbers of order 100. These results confirm the stabilizing role of the magnetic field and the either stabilizing or destabilizing role of the electric field, depending on its orientation in the spanwise direction. They can be considered an improvement of those obtained by the averaged model used in the previous related works.

\section{APPENDIX A}

Setting the total field $\mathbf{B}$ as the sum of $\mathbf{B}_{\mathbf{0}}$ and the induced field, namely, $\mathbf{B}=\mathbf{B}_{\mathbf{0}}+\operatorname{Re}_{m} \mathbf{b}$, where $\operatorname{Re}_{m}=\mu_{0} \sigma u_{0} h_{0}$ $\left(\mathrm{Re}_{m} \ll 1\right)$ denotes the magnetic Reynolds number, and setting $\mathbf{b}=\left(b_{1}, \epsilon b_{2}, 0\right)^{t}$, the coupled dimensionless MHD equations read, up to $O\left(\epsilon^{2}, R_{m}\right)$,

$$
\begin{gathered}
b_{1 y y}+\sin \theta u_{y}+\epsilon \cos \theta u_{x}+\epsilon^{2} b_{1 x x}=0 \\
b_{2 y y}+\sin \theta v_{y}+\epsilon \cos \theta v_{x}+\epsilon^{2} b_{2 x x}=0 \\
b_{1 x}+b_{2 y}=0 \\
u_{x}+v_{y}=0, \\
\operatorname{Re} \epsilon\left(u_{t}+u u_{x}+v u_{y}\right)=-\left(p+\epsilon \mathrm{Ha}^{2} \widetilde{B}\right)_{x}+u_{y y}+3 \\
+\epsilon^{2} u_{x x}+\mathrm{Ha}^{2} B_{y}, \\
0=-\left(p+\epsilon \mathrm{Ha}^{2} \widetilde{B}\right)_{y}-3 \epsilon \cot \beta+\epsilon^{2}\left(v_{y y}-\mathrm{Ha}^{2} B_{x}\right),
\end{gathered}
$$

where the pressure is scaled by $\rho u_{0}^{2} / \operatorname{Re} \epsilon$ and $B$ and $\tilde{B}$ are defined as

$$
\begin{aligned}
& B=\sin \theta b_{1}-\epsilon \cos \theta b_{2}, \\
& \widetilde{B}=\cos \theta b_{1}+\epsilon \sin \theta b_{2} .
\end{aligned}
$$

Equations (A1)-(A6) are subject to appropriate boundary conditions, which will be specified below; they are complemented by the dimensionless version of Eqs. (5)-(7),

$$
\begin{gathered}
\boldsymbol{j}=\boldsymbol{\nabla} \times \mathbf{b}, \\
\delta \boldsymbol{E}=\boldsymbol{j}-\boldsymbol{v} \times \boldsymbol{B}, \\
\nabla \times \mathbf{E}=-\frac{\epsilon \operatorname{Re}_{m}}{\delta} \frac{\partial \mathbf{b}}{\partial t} .
\end{gathered}
$$

First, observing that $\boldsymbol{\nabla} \cdot \mathbf{E}=0$ (since $\nabla \cdot \mathbf{j}=0$ and $\mathbf{B} \cdot \nabla \times$ $\mathbf{v}=0)$ and $\nabla \times \mathbf{E}=O\left(\epsilon \mathrm{Re}_{m}\right)$, the induced electric field is $O\left(\epsilon \mathrm{Re}_{m}\right)$ and therefore can be neglected. So $\mathbf{E}$ remains unchanged up to our approximation. 
Now, eliminating the total pressure by cross differentiation of (A5) and (A6), one obtains

$$
\begin{aligned}
& \operatorname{Re} \epsilon\left(u_{t}+u u_{x}+v u_{y}\right)_{y} \\
& =u_{y y y}+2 \epsilon^{2} u_{x x y}+\operatorname{Ha}^{2}\left(B_{y y}+\epsilon^{2} B_{x x}\right),
\end{aligned}
$$

where the magnetic part can be eliminated in favor of the velocity. Indeed, by combining (A1) and (A2), we are led to

$$
B_{y y}+\epsilon^{2} B_{x x}=\epsilon \sin 2 \theta v_{y}+\epsilon^{2} \cos ^{2} \theta v_{x}-\sin ^{2} \theta u_{y} .
$$

So (A12) takes the form

$$
\begin{aligned}
\operatorname{Re} \epsilon\left(u_{t}+u u_{x}+v u_{y}\right)_{y}= & u_{y y y}+2 \epsilon^{2} u_{x x y}+\operatorname{Ha}^{2}\left(\epsilon \sin 2 \theta v_{y}\right. \\
& \left.+\epsilon^{2} \cos ^{2} \theta v_{x}-\sin ^{2} \theta u_{y}\right) .
\end{aligned}
$$

Equations (A4) and (A14) must be solved, subject to impermeability and no slip conditions at the wall, $\left.u\right|_{y=0}=\left.v\right|_{y=0}=0$, the kinematic free surface condition, which can be written in its global form

$$
q_{x}+h_{t}=0,
$$

and normal and tangential stress balances at the free surface,

$$
\begin{aligned}
& u_{y}=\epsilon^{2}\left(4 u_{x} h_{x}-v_{x}\right), \\
& p=2 \epsilon^{2} v_{y}-W \epsilon h_{x x} .
\end{aligned}
$$

We observe that the magnetic and electric fields do not occur in the free surface stress balances since $\mathrm{Re}_{m}$ is small and due to the continuity of the normal magnetic and electric field components. Integrating (A14) with respect to $y$ and using wall conditions give

$$
\begin{aligned}
\operatorname{Re} \epsilon & \left(u_{t}+u u_{x}+v u_{y}\right) \\
= & u_{y y}-\left.u_{y y}\right|_{y=0}+2 \epsilon^{2} u_{x x} \\
& +\mathrm{Ha}^{2}\left(\epsilon v \sin 2 \theta-u \sin ^{2} \theta+\epsilon^{2} \cos ^{2} \theta \int_{0}^{y} v_{x} d y\right) .
\end{aligned}
$$

The integration constant $\left.u_{y y}\right|_{y=0}$ can be deduced from the projection of the $x$-momentum equation (A5) on $y=0$; we get

$$
\left.u_{y y}\right|_{y=0}=\left.p_{x}\right|_{y=0}-3-\left.\mathrm{Ha}^{2} \sin \theta b_{1 y}\right|_{y=0} .
$$

Equation (A9) shows that $\left.b_{1 y}\right|_{y=0}$ is, up to a sign, nothing more than the $z$ component of the current density. So, from (A10), one obtains $\left.b_{1 y}\right|_{y=0}=-\delta$ and therefore

$$
\left.u_{y y}\right|_{y=0}=\left.p_{x}\right|_{y=0}-3+\delta \mathrm{Ha}^{2} \sin \theta,
$$

where the pressure gradient along the plate can be calculated from the pressure distribution across the fluid layer. For this, let us first observe that the normal pressure gradient can be cast in the form

$$
p_{y}=\epsilon^{2} v_{y y}-3 \epsilon \cot \beta-\epsilon \mathrm{Ha}^{2} \cos \theta b_{1 y},
$$

where $b_{1 y}$ is obtained by integrating (A1) with respect to $y$, namely,

$$
b_{1 y}=-\delta+\epsilon v \cos \theta-u \sin \theta .
$$

Now, integrating (A21) through the film depth and using the normal stress balance at the free surface yield the pressure distribution

$$
p(x, y, t)=\epsilon^{2}\left(v_{y}+\left.v_{y}\right|_{h}\right)-W \epsilon h_{x x}+3 \epsilon \cot \beta(h-y)-\epsilon \mathrm{Ha}^{2} \cos \theta\left\{\epsilon \cos \theta \int_{h}^{y} v d y-\sin \theta \int_{h}^{y} u d y-\delta(y-h)\right\} .
$$

Then, expressing the streamwise pressure gradient at the wall allows us to transform (A18) into

$$
\begin{aligned}
\operatorname{Re} \epsilon\left(u_{t}+u u_{x}+v u_{y}\right)= & u_{y y}+3-\mathrm{Ha}^{2} \sin \theta(\delta+u \sin \theta)+\epsilon\left\{W h_{x x x}-3 \cot \beta h_{x}+\mathrm{Ha}^{2} \cos \theta\left[\sin \theta\left(2 v-\left.v\right|_{h}\right)\right.\right. \\
& \left.\left.+h_{x}\left(\delta+\left.\sin \theta u\right|_{h}\right)\right]\right\}+\epsilon^{2}\left\{2 u_{x x}+\left(\left.u_{x}\right|_{h}\right)_{x}+\mathrm{Ha}^{2} \cos ^{2} \theta\left[\int_{h}^{y} v_{x} d y-\left.v\right|_{h} h_{x}\right]\right\}
\end{aligned}
$$

\section{APPENDIX B}

Expressions of the coefficients of the trial functions and the first order correction of the velocity profile.

$$
\begin{aligned}
f_{1}^{*}(\xi) & =\frac{1}{2 \mathrm{Ha}^{3}}\left(\xi \sinh \mathrm{Ha} \xi-h \frac{\tanh \mathrm{Ha} h}{\cosh \mathrm{Ha} h} \cosh \mathrm{Ha} \xi\right), \\
f_{2}^{*}(\xi) & =\frac{1}{4 \mathrm{Ha}^{4}}\left\{\left(\xi^{2}+5 \frac{h}{\mathrm{Ha}} \tanh \mathrm{Ha} h-h^{2}\right) \cosh \mathrm{Ha} \xi-\frac{5}{\mathrm{Ha}} \xi \sinh \mathrm{Ha} \xi\right\}, \\
\delta_{1}(x, t) & =\frac{1}{4 \mathrm{Ha}^{2}} \frac{(1+4 \mathrm{Ha} h) \cosh \mathrm{Ha} h+(4 \mathrm{Ha} h-1) \sinh \mathrm{Ha} h-(\cosh 3 \mathrm{Ha} h+\sinh 3 \mathrm{Ha} h)}{\cosh 2 \mathrm{Ha} h+\sinh 2 \mathrm{Ha} h+\mathrm{Ha} h(\cosh 2 \mathrm{Ha} h-\sinh 2 \mathrm{Ha} h-1)-1}, \\
\delta_{2}(x, t) & =\frac{2 \mathrm{Ha}(\sinh 3 \mathrm{Ha} h+11 \sinh \mathrm{Ha} h)+(2 \mathrm{Ha} h-7) \cosh 3 \mathrm{Ha} h+(26 \mathrm{Ha} h+7) \cosh \mathrm{Ha} h}{8 H a^{4}(\mathrm{Ha} h-1)(\cosh 2 \mathrm{Ha} h+\sinh 2 \mathrm{Ha} h)+8 \mathrm{Ha}^{4}(1+\mathrm{Ha} h)} . \\
\alpha_{1}(x, t) & =\frac{\mathrm{Ha}^{5} q h\left(\cosh ^{2} \mathrm{Ha} h-\mathrm{Ha} h \sinh \mathrm{Ha} h \cosh \mathrm{Ha}_{+}+1\right)}{\sinh \mathrm{Ha} h\left[1-\cosh ^{2} \mathrm{Ha} h\left(1+3 h^{2} \mathrm{Ha}^{2}\right)\right]+\mathrm{Ha} h \cosh \mathrm{Ha} h\left[\cosh ^{2} \mathrm{Ha} h\left(3+h^{2} \mathrm{Ha}^{2}\right)-3\right]},
\end{aligned}
$$




$$
\begin{aligned}
& \alpha_{2}(x, t)=\frac{\mathrm{Ha}^{5} q^{2}\left(1-\cosh ^{2} \mathrm{Ha} h+\mathrm{Ha} h \sinh \mathrm{Ha} h \cosh \mathrm{Ha} h\right)}{\sinh \mathrm{Ha} h\left[1-\cosh ^{2} \mathrm{Ha} h\left(1+3 h^{2} \mathrm{Ha}^{2}\right)\right]+\mathrm{Ha} h \cosh \mathrm{Ha} h\left[\cosh ^{2} \mathrm{Ha} h\left(3+h^{2} \mathrm{Ha}^{2}\right)-3\right]}, \\
& \alpha_{3}(x, t)=\frac{\left[1-\cosh ^{2} \mathrm{Ha} h-h^{2} \mathrm{Ha}^{2} \cosh (\mathrm{Ha} h)^{2}+2 h \mathrm{Ha} \cosh \mathrm{Ha} h \sinh \mathrm{Ha} h\right] \mathrm{Ha}^{3}}{\operatorname{sinh~Ha} h\left[1-\cosh ^{2} \mathrm{Ha} h\left(1+3 h^{2} \mathrm{Ha}^{2}\right)\right]+\mathrm{Ha} h \cosh \mathrm{Ha} h\left[\cosh ^{2} \mathrm{Ha} h\left(3+h^{2} \mathrm{Ha}^{2}\right)-3\right]}, \\
& \beta_{1}(x, t)=\frac{\mathrm{Ha}^{6} q \cosh \mathrm{Ha} h(\sinh \mathrm{Ha} h-\mathrm{Ha} h \cosh \mathrm{Ha} h)}{\operatorname{sinh~Ha} h\left[1-\cosh ^{2} \mathrm{Ha} h\left(1+3 h^{2} \mathrm{Ha}^{2}\right)\right]+\mathrm{Ha} h \cosh \mathrm{Ha} h\left[\cosh ^{2} \mathrm{Ha} h\left(3+h^{2} \mathrm{Ha}^{2}\right)-3\right]}, \\
& \beta_{2}(x, t)=\frac{\mathrm{Ha}^{7} q^{2}\left(\cosh ^{2} \mathrm{Ha} h-1\right)}{\operatorname{sinh~Ha} h\left[1-\cosh ^{2} \mathrm{Ha} h\left(1+3 h^{2} \mathrm{Ha}^{2}\right)\right]+\mathrm{Ha} h \cosh \mathrm{Ha} h\left[\cosh ^{2} \mathrm{Ha} h\left(3+h^{2} \mathrm{Ha}^{2}\right)-3\right]} .
\end{aligned}
$$

\section{APPENDIX C}

Following common practice, we decompose the velocity and magnetic fields into the base states $\left(u_{b}, 0\right)$ and $(\cos \theta, \sin \theta)$ and two-dimensional disturbances $(u, v)$ and $\left(b_{1}, b_{2}\right)$, and the momentum OS equations read

$$
\begin{gathered}
\operatorname{Re}\left[u_{t}+u_{b} u_{x}+v u_{b y}\right]=-p_{x}+\nabla^{2} u+\mathrm{Ha}^{2} \sin \theta\left(b_{1 y}-b_{2 x}\right), \\
\operatorname{Re}\left[v_{t}+u_{b} v_{x}\right]=-p_{y}+\nabla^{2} v+\mathrm{Ha}^{2} \cos \theta\left(b_{2 x}-b_{1 y}\right) .
\end{gathered}
$$

Then, introducing the stream function $\psi(x, y, t)=\phi(y) e^{i \alpha(x-c t)}$ and using Eqs. (A1)-(A3) to eliminate the induced magnetic field in favor of the velocity field lead to a linear set of equations which can be put in the following compact form:

$$
\frac{d}{d t}\left(\phi, \phi^{\prime}, w, p^{*}\right)^{t}=\left[\begin{array}{ll}
O_{1} & O_{2} \\
O_{3} & O_{4}
\end{array}\right]\left(\phi, \phi^{\prime}, w, p^{*}\right)^{t},
$$

with

$$
\begin{array}{ll}
O_{1}=\left[\begin{array}{cc}
0 & 1 \\
-\alpha^{2} & 0
\end{array}\right], \quad O_{2}=\left[\begin{array}{ll}
0 & 0 \\
1 & 0
\end{array}\right], & O_{4}=i \alpha\left[\begin{array}{ll}
0 & 1 \\
1 & 0
\end{array}\right], \\
O_{3}=\left[\begin{array}{cc}
i \alpha\left[\frac{1}{2} \mathrm{Ha}^{2} \sin 2 \theta-\operatorname{Re} u_{b}^{\prime}\right] & \mathrm{Ha}^{2} \sin ^{2} \theta-i \alpha \operatorname{Re}\left(c-u_{b}\right)+4 \alpha^{2} \\
\left.\alpha^{2} \operatorname{Re}\left(c-u_{b}\right)+i \alpha \mathrm{Ha}^{2} \cos ^{2} \theta\right) & \frac{1}{2} \mathrm{Ha}^{2} \sin 2 \theta
\end{array}\right],
\end{array}
$$

where $w=\phi^{\prime \prime}+\alpha^{2} \phi$ is the stress tensor component $\tau_{x y}, p^{*}$ is the modified pressure introduced for convenience through the transformation $p^{*}=p+2 u_{x}$, and the primes indicate differentiation with respect to $y$. The system (C3) is subject to the boundary conditions

$$
\begin{gathered}
\left.\phi\right|_{0}=\left.\phi^{\prime}\right|_{0}=0, \\
{\left[w\left(c-u_{b}\right)+u_{b}^{\prime \prime} \phi\right]_{y=1}=0,} \\
\left(u_{b}-c\right) p^{*}+\left.\left(\alpha^{2} W+G \cot \beta-\delta \mathrm{Ha}^{2} \cos \theta\right) \phi\right|_{y=1}=0 .
\end{gathered}
$$

In order to seek a numerical solution to the OS problem, the so-called Riccati matrix $\mathcal{R}$ is introduced through the transformation $\left(\Phi, \Phi^{\prime}\right)^{t}=\mathcal{R}\left(w, p^{*}\right)^{t}$, which allows us to transform the boundary eigenvalue problem (the complex phase velocity $c$ is regarded as the eigenvalue parameter) into the following nonlinear initial value problem:

$$
\begin{aligned}
\frac{d}{d y} \mathcal{R} & =-\mathcal{R} O_{3} \mathcal{R}-\mathcal{R} O_{4}+O_{1} \mathcal{R}+O_{2}, \\
\mathcal{R}(0) & =0 .
\end{aligned}
$$

This problem does not suffer from the parasitic growth problem encountered when using the shooting method. It has been successfully applied by Davey [33] in the hydrodynamic case. On solving (C7a) and (C7b) by a fourth order Runge-Kutta scheme, the phase velocity must be varied until the boundary conditions at $y=1$ are satisfied. An eigenvalue equation is then deduced in the form

$$
\operatorname{det}\left\{\left(\begin{array}{cc}
\frac{u_{b}^{\prime \prime}(1)}{c-u_{b}(1)} & 0 \\
\frac{\delta \mathrm{Ha}^{2} \cos \theta-\alpha^{2} W-G \cot \beta}{c-u_{b}(1)} & 0
\end{array}\right) \mathcal{R}+\left(\begin{array}{ll}
1 & 0 \\
0 & 1
\end{array}\right)\right\}=0 .
$$


[1] P. L. Kapitza and S. P. Kapitza, Sov. Phys. J. Exp. Theor. Phys. 19, 105 (1949).

[2] A. E. Dukler, Chem. Eng. Educ. 11, 108 (1976).

[3] P. N. Yoshimura, T. Nosoko, and T. Nagata, Chem. Eng. Sci. 51, 1231 (1996).

[4] S. J. Weinstein and K. J. Ruschak, Annu. Rev. Fluid Mech. 36, 29 (2004).

[5] C.-S. Yih, Quart. Appl. Math. 12, 434 (1955).

[6] T. B. Benjamin, J. Fluid Mech. 2, 554 (2006).

[7] D. J. Benney, J. Math. Phys. 45, 150 (1966).

[8] S. V. Alekseenko, V. E. Nakoryakov, and B. G. Pokusaev, AIChE J. 31, 1446 (1985).

[9] T. Ooshida, Phys. Fluids 11, 3247 (1999).

[10] V. Ya. Shkadov, Izv. Akad. Nauk SSSR Mekh. Zhidk. Gaza 2, 43 (1967)

[11] C. Ruyer-Quil and P. Manneville, Eur. Phys. J. B 6, 277 (1998).

[12] C. Ruyer-Quil and P. Manneville, Eur. Phys. J. B 15, 357 (2000).

[13] C. Ruyer-Quil and P. Manneville, Phys. Fluids 14, 170 (2002).

[14] C. Ruyer-Quil, B. Scheid, S. Kalliadasis, M. G. Velarde, and R. Kh. Zeytounian, J. Fluid Mech. 538, 199 (2005).

[15] B. Scheid, C. Ruyer-Quil, S. Kalliadasis, M. G. Velarde, and R. Kh. Zeytounian, J. Fluid Mech. 538, 223 (2005).

[16] M. Amaouche, N. Mehidi, and N. Amatousse, Phys. Fluids 19, 084106 (2007).

[17] S. J. D. D'Alessio, J. P. Pascal, H. A. Jasmine, and K. A. Ogden, J. Fluid Mech. 665, 418 (2010).
[18] P. Pascal and S. J. D. D’Alessio, Int. J. Multiphase Flow 36, 449 (2010).

[19] H. B. Löfgren and H. O. Åkerstedt, Fluid Dyn. Res. 23, 1 (1998).

[20] V. A. Glukhikh, A. V. Tananaev, and I. R. Kirillov, Magnetohydrodynamics in Nuclear Power (Énergoatomizdat, Moscow, 1987) (in Russian).

[21] A. Mukaopadhyay and B. S. Dandapat, J. Phys. D: Appl. Phys. 38, 138 (2005).

[22] A. González and A. Castellanos, Phys. Rev. E 53, 3573 (1996).

[23] D. Tseluiko and D. T. Papageorgiou, J. Fluid Mech. 556, 361 (2006).

[24] B. Tileey, P. Petropoulos, and D. T. Papageorgiou, Phys. Fluids 13, 3547 (2001).

[25] D. T. Papageorgiou and J. M. Vanden-Broeck, J. Fluid Mech. 508, 71 (2004).

[26] D. T. Papageorgiou and J. M. Vanden-Broeck, Eur. J. Appl. Math. 15, 609 (2004).

[27] S. Korsunsky, Eur. J. Mech. B 18, 295 (1999).

[28] P. C. Lu and G. S. R. Sarma, Phys. Fluids 10, 2339 (1967).

[29] A. Mukhopadhyay, B. S. Dandapat, and A. Mukhopadhyay, Int. J. Nonlinear Mech. 43, 632 (2008).

[30] H. Ait Abderrahmane and G. H. Vatistas, Fusion. Eng. Des. 83, 661 (2008).

[31] See Supplemental Material at http://link.aps.org/supplemental/ 10.1103/PhysRevE.88.023028 for Maple scripts for the derivation of the reduced models.

[32] M. Amaouche, A. Djema, and H. Ait Abderrahmane, Eur. J. Mech. B 34, 70 (2012).

[33] A. Davey, J. Comput. Phys. 24, 331 (1977). 